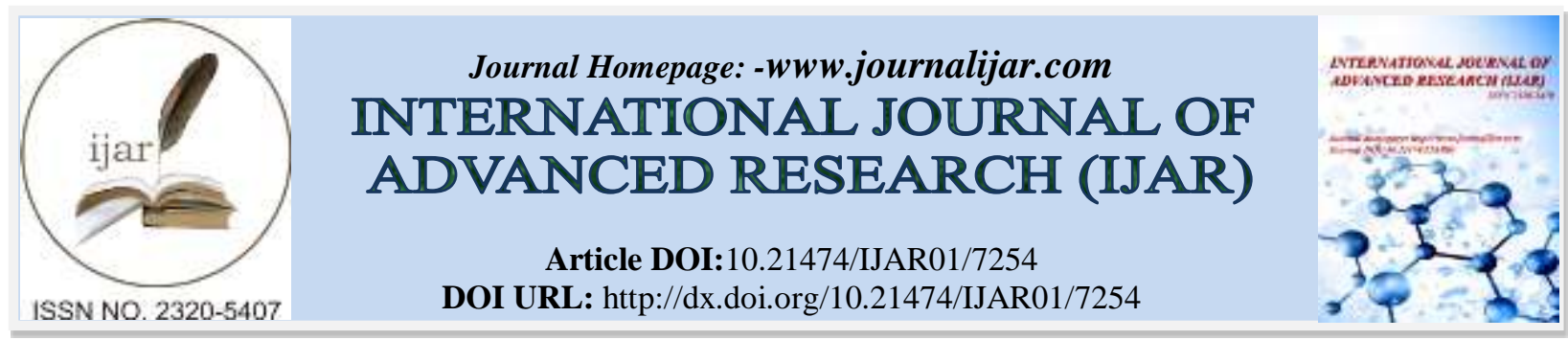

RESEARCH ARTICLE

\title{
IMMEDIATE POST-EXTRACTION DENTAL IMPLANTS WITH AND WITHOUT BONE GRAFTING MATERIALS: SYSTEMATIC REVIEW.
}

\section{Huda Hamed Basher Mohamed ${ }^{1}$ and Asma M. Serag Eldien ${ }^{2}$.}

1. (M.S.D) PhD Degree candidate, Department of Oral Medicine, Oral Diagnosis and Periodontology, Faculty of Oral and Dental Medicine, Cairo University. Assistant lecturer, Department of Periodontology,Benghazi University.

2. (M.S.D, Ph.D) Lecturer, Department of Periodontology, 6 October University.

\section{Manuscript Info}

-........................

Manuscript History

Received: 10 April 2018

Final Accepted: 12 May 2018

Published: June 2018

Keywords:-

Systematic review, randomized controlled trials, Dental Implants, Bone Transplantation, Bone Grafting.

\section{Abstract}

Aim: To compare the stability and survival rate of the immediate dental implants that are placed in the fresh extraction sockets with and without bone grafts.

Methods: An electronic search in MEDLINE (PubMed) and the Cochrane Library were performed to include randomized controlled trials on immediate implants with a mean follow-up time of at least 6 months. We hand searched citation lists of relevant publications. No language or date restriction was applied. Independently the authors screened, reviewed and assessed the studies for the risk of bias. This was followed by data extraction and analysis of different outcomes.

Results: We included two studies (68 participants). The two studies reported no implant failures after 6 months follow up period, so no difference in the implant survival between the two groups (RR 1.00, $95 \%$ CI, 0.92 to 1.08). One trial reported the bone density after 6 months follow up. There is significant difference in bone density favor the use of bone grafting materials group (RR 245.00, 95\% CI, 38.51 to 451.49) and one trial reported the bucco-lingual crestal width with significant difference in favor the use of bone grafting materials group $(1.31,95 \% \mathrm{CI}, 0.38$ to 2.23$)$. No trial evaluated the implant survival rate, bone density and bucco-lingual crestal width after one year. Implant stability, patient satisfaction and the post-surgical marginal bone loss were not reported on the included trials.

Conclusions: There is inadequate evidence to suggest the bone grafts with immediate post-extraction single tooth implants to enhance implant stability and survival rate.

Copy Right, IJAR, 2018,. All rights reserved.

\section{Introduction:-}

Tooth extraction is one of the most common dental procedures. Alveolar bone loss after tooth extraction is an inevitable process and can prevent favorable position and angulation of implant placement (Cardaropoli et al., 2003, Araujo et al., 2005).

After tooth extraction the bone remodeling, in the anterior maxilla at the facial bone will resorb up to $25 \%$ during the first year and up to $40 \%$ to $60 \%$ over the three years later. In the posterior region, the rate of bone loss is greater 
reaching up to $50 \%$ within the first year after extraction, which reducing the available bone for placement of implants (Mish and Judy, 2000). And Christensen (1996), reports an annual resorption rate of at least $0.5 \%$ to $1 \%$ during the remainder of a Patient's life. Maintenance of bone contour for dental implants, pontic design, dental appliance stability, soft tissue aesthetics and maintaining the gum condition of adjacent teeth are necessary considerations following an extraction.

Immediate placement of implant in fresh extraction sockets have many benefits over Branemark's protocol for conventional implant placement: total treatment time and number of surgical procedures is reduced, additional ideal implant positioning is possible, soft tissue height and contour are preserved within the esthetic zone, opportunities for osseointegration are better due to healing potential of recent extraction socket (Schwart et al., 2000).

The dilemma facing implants placed in fresh extraction sockets has been the horizontal distance between the implant surface and the labial plate of bone (the jumping gap). Clinical healing appears to be non-eventful in most cases, but the question remains as to the interface of implant and bone. It has been postulated that a connective tissue interface can develop once the gap is larger than $1.5 \mathrm{~mm}$ at the coronal aspect of the implant (Knox et al., 1991) while some suggest Botticelli et al; (2003), the space should be $0.5 \mathrm{~mm}$ or less. To achieve both functional and aesthetic requirements, it might be necessary to plan implant placement after tooth extraction and socket grafting/ridge preservation. Bone graft materials have played on important role in periodontal regeneration for several years (Hoexter, 2002). To maintain alveolar bone width and height for implant placement or for prosthetic concerns, allografts, xenografts and alloplasts have been used to graft extraction sites.

Bone grafts are classified on the basis of how they produce new bone: osteoconduction, osteoinduction, and/or osteogenesis. Osteoconduction happens once the bone graft material serves as a scaffold for new bone growth by the native bone. In the very least, a bone graft material should be osteoconductive. Osteoinduction involves the stimulation of osteoprogenitor cells to differentiate into osteoblasts that then begin new bone formation. Osteogenesis happens once vital osteoblasts originating from the bone graft material contribute to new bone growth along with bone growth generated via the other two mechanisms (Misch and Dietsh 1993, Klokkevold and Jovanovic 2002). All grafting materials have one or more of these three mechanisms of action.

Several approaches for augmenting post-extractive sites are currently used and a few of those are evaluated in randomized controlled trials (RCTs). However, it is still unclear if these approaches are required and which could be the most effective augmentation technique (De Angelis et al., 2011).

The aim of the review was to compare the stability and survival rate of the immediate dental implants that are placed in the fresh extraction sockets with and without bone grafts.

\section{Focused question:-}

In patients with irrestorable tooth what is the effect of using bone graft materials or not in conjunction with immediate implant on the stability and survival rate of implant?

\section{Methodology:-}

In this meta- Meta-Analysis, only randomized controlled trials, more than 6 months follow-up, implant details described, 10 or more implant sample were included. The patients were rehabilitated with immediate post extraction single tooth implant in healthy socket (Both arches, all sites and All implant loading protocols were included). The comparisons were performed between immediate dental implants with no bone grafting materials versus with bone grafting materials.

\section{Electronic search:-}

An electronic search in PubMed and the Cochrane Library were performed to incorporate randomized controlled trials on immediate implants with a mean follow-up time of a minimum of 6 months. The last electronic search was conducted on April 2018. We hand searched citation lists of relevant publications. No language or date restriction was applied. 
The search strategy used was a combination of Mesh terms and text words:

Randomized controlled trial [Publication Type] OR

Controlled clinical trial [Publication Type] OR

Randomized[Title/Abstract] OR

Randomly[Title/Abstract] OR

Trial[Title/Abstract] OR

Groups[Title/Abstract] NOT

Animals[MeSH Terms] NOT humans[MeSH Terms]

AND

Dental Implants [MeSH Terms] OR

Dental Implants [Title/Abstract] OR

AND

Bone Transplantation [MeSH Terms] OR

Bone Transplantation [Title/Abstract] OR

Bone Grafting [Title/Abstract]

\section{Study Selection:-}

After the electronic search, two free reviewers (H.H.M. and A.M.S. screened all titles and decided the amount of abstracts to be assessed. Following this, the two independent reviewers screened every single chosen for possible inclusion within the review and decided the decision of full-content articles. The full texts of all papers of possible relevance were then obtained for free evaluation by the reviewers. Any contradiction was resolved by discussion.

\section{Investigation outcomes:-}

The primary outcome was implant survival rate, implant stability while the secondary outcome was the bone density, the bucco-lingual crestal width, Patient satisfaction and the post-surgical marginal bone loss at (6-12) months and more than one year.

\section{Risk of bias assessment:-}

Two review authors free assessed risk of bias for each study using the randomized controlled trial checklist of the Cochrane Center, CONSORT guidelines. The following seven criteria were used: selection bias, allocation bias, performance bias, detection bias, defined inclusion/exclusion criteria, attrition bias and reporting bias. They made express judgments about whether the papers are at "Low risk," "High risk," or "Unclear risk" of bias, according to the criteria given in the Handbook (Higgins JPT, 2011). There were no contradictions on the appraisal of risk of bias of included articals.

\section{Data analysis and synthesis:-}

The statistical analysis performed by using the Review Manager software (The Nordic Cochrane Centre, 2014). For dichotomous data, we presented results as summary risk ratio with $95 \% \mathrm{CI}$ and for continuous data; we used the mean difference with 95\% CI. The statistical unit was the implants in each group in all the outcomes. The statistical heterogeneity in each meta-analysis was assessed by using the $\mathrm{T}^{2}, \mathrm{I}^{2}$ and $\mathrm{Chi}^{2}$ statistics. The heterogeneity as substantial was regarded, if $\mathrm{I}^{2}$ was greater than $30 \%$ and either $\mathrm{T}^{2}$ was greater than zero, or there was a low $\mathrm{P}$ value (less than 0.10) in the $\mathrm{Chi}^{2}$ test for heterogeneity. The fixed-effect meta-analysis for combining data was used where it is reasonable to assume that studies were estimating the same underlying treatment effect: i.e. where trials were examining the same intervention, and we judged the trials' populations and methods sufficiently similar. If there was clinical heterogeneity sufficient to expect that the underlying treatment effects differed between trials, or if the substantial statistical heterogeneity was detected, the sensitivity analysis was explored this followed by randomeffects if required.

\section{Results:-}

Two studies (68 participants) were included in this review. (Bottini LP et al., 2012) (Daif, 2013). Two studies(Chen et al., 2005)(Chen and Darby, 2007) were excluded which they enclosed patients with infected sockets restored by immediate post extraction implant.

All included patients were restored with immediate post-extraction single tooth implants and randomised to with bone grafting material versus no bone grafting material. 
Bottini et al., (2012) included patients receiving single implants placed into immediate extraction sockets of a molar or a first upper premolar with a flapless approach. In the control sites no grafting material was placed, while in the test sites a deantigenated collagenated bone substitute of porcine origin was used to fill the gaps between the implant and the extraction socket.

Daif (2013) included patients indicated for extraction of their lower premolars and insertion of immediate dental implants. They were randomly divided into two groups. Group A received immediate dental implants without any filling material around the implants, while in group B, a pure-phase multiporous beta-TCP was gently packed into the bone gaps around the implants.

\section{Risk of bias in included studies:-}

The two included studies represented sufficient techniques of sequence generation (Bottini et al., 2012) (Daif. 2013) but no data on how the sequence was concealed. (Bottini et al., 2012) was assessed as high risk of attrition bias due to performing per-protocol analysis. The other trial included all randomized participants and was at low risk of attrition bias. The two included trials were at high risk of reporting bias. Bottini et al., (2012), Daif (2013) neglected to incorporate outcomes for key results anticipated that would have been reported for such studies. See fig. $(2,3)$.

\section{Primary outcome:-}

Implant survival rate:-

Bottini et al., (2012) and Daif (2013) reported successful all implants in both groups and no implant failures after a follow up period after 6 months, so no difference in the implant survival between the two groups see (fig. 3) (RR $1.00,95 \% \mathrm{CI}, 0.92$ to 1.08$)$. No trial evaluated the implant survival rate after one year.

\section{Implant stability:-}

None of the included studies reported this outcome

\section{Secondary outcomes:-}

The bone density:-

This outcome was only reported by Daif (2013). There is significant difference in bone density favor the use of bone grafting materials with immediate post-extraction implant see figure (4) (RR 245.00, 95\% CI, 38.51 to 451.49).

\section{The bucco-lingual crestal width:-}

Bottini et al. 2012 reported this outcome and there is significant difference in buccolingual crestal width favor the use of bone grafting materials with immediate post-extraction implant see figure (5) (1.31, 95\% CI, 0.38 to 2.23 ).

Patient satisfaction and the post-surgical marginal bone loss were not evaluated on the two included trials. 
Fig. 1:-PRISMA flow diagram

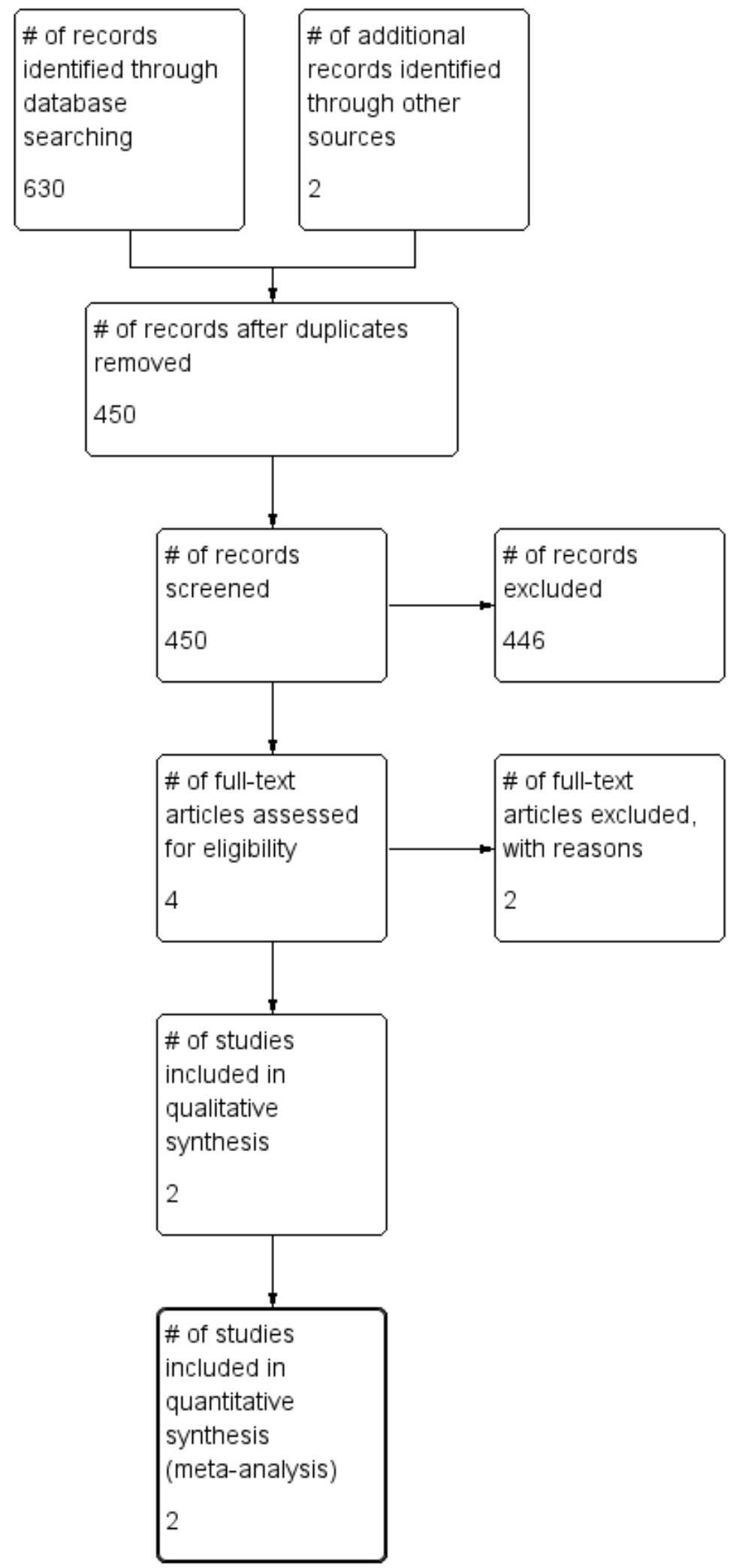


Fig. 2:-Risk of bias summary: review authors' judgements about each risk of bias item for each included study.

\begin{tabular}{|c|c|c|c|c|c|c|c|c|}
\hline & 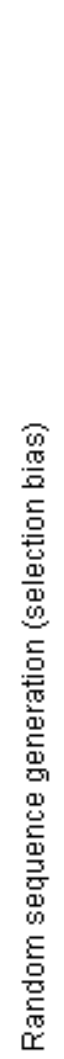 & 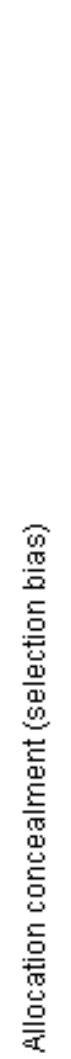 & 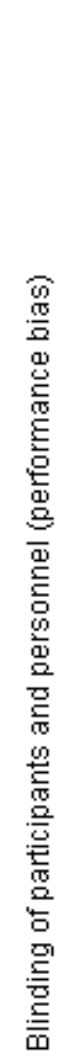 & 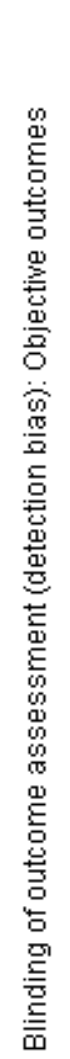 & 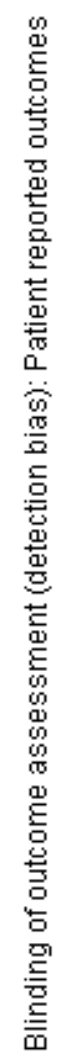 & 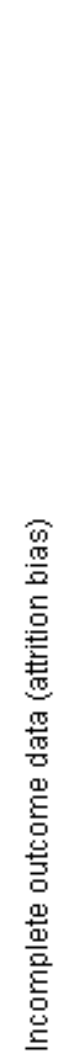 & 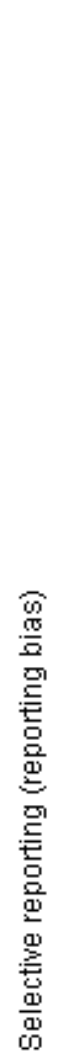 & 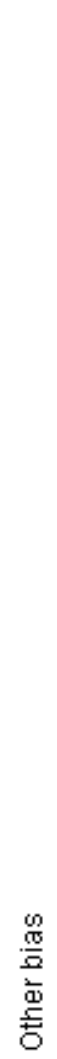 \\
\hline Bottini 2012 & + & $?$ & + & + & & & & + \\
\hline Daif 2013 & + & $?$ & + & + & & + & $\odot$ & + \\
\hline
\end{tabular}

Fig. 3:-Risk of bias graph: review authors' judgements about each risk of bias item presented as percentages across all included studies

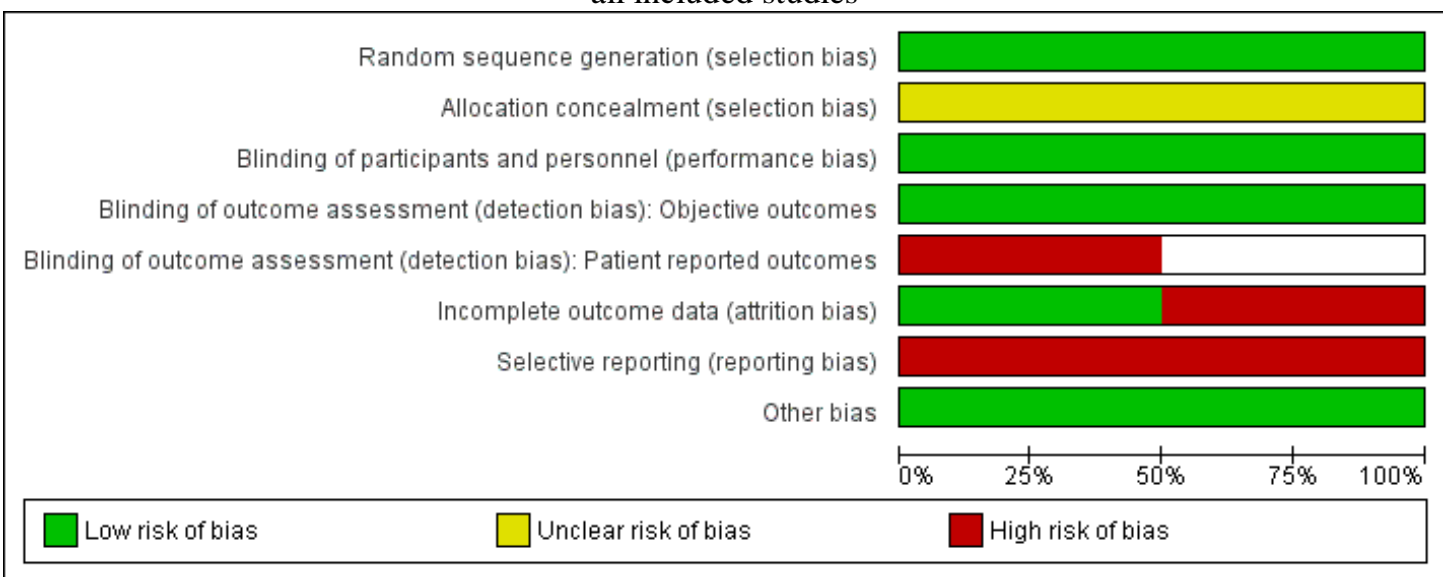




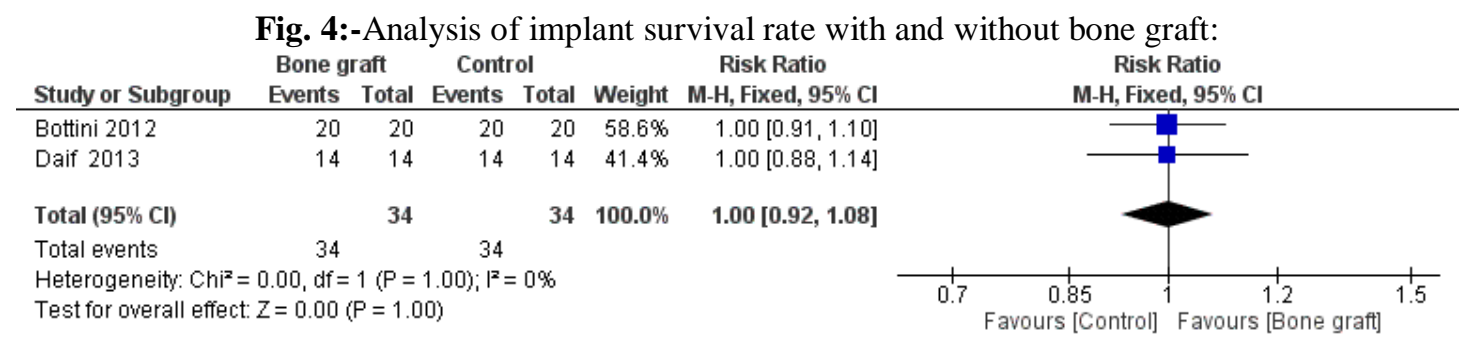

Fig. 5:-Analysis of bone density around the implant with and without bone graft:

\begin{tabular}{|c|c|c|c|c|c|c|c|c|c|c|c|c|}
\hline Study or Subgroup & \multicolumn{3}{|c|}{ Bone graft } & \multicolumn{3}{|c|}{ Control } & \multicolumn{2}{|r|}{ Mean Difference } & \multicolumn{3}{|c|}{$\begin{array}{l}\text { Mean Difference } \\
\text { IV, Fixed, } 95 \% \mathrm{Cl}\end{array}$} & \\
\hline Daif 2013 & 1,490 & 358 & 14 & 1,245 & 165 & 14 & $100.0 \%$ & $245.00[38.51,451.49]$ & & & & \\
\hline Total $(95 \% \mathrm{Cl})$ & & & 14 & & & 14 & $100.0 \%$ & $245.00[38.51,451.49]$ & & & & \\
\hline $\begin{array}{l}\text { Heterogeneity: Not a } \\
\text { Test for overall effec }\end{array}$ & $\begin{array}{l}\text { plicable } \\
Z=2.33\end{array}$ & $(\mathrm{P}=$ & .02) & & & & & & -50 & $\begin{array}{r}-250 \\
\text { urs }[\mathrm{C}\end{array}$ & $\begin{array}{lr}0 & 2 \\
1] & \text { Favours }\end{array}$ & $\begin{array}{lr}250 & 500 \\
s\end{array}$ \\
\hline
\end{tabular}

Fig. 6:-Analysis of bucco-lingual crestal width at area of implant with and without bone graft:

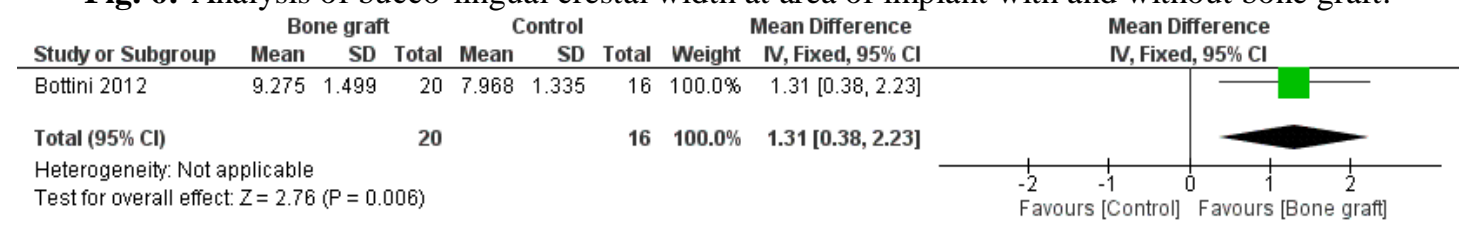

\section{Discussion:-}

This systematic review was included two randomized control trails with (48 participants) that evaluated the efficacy of bone graft versus no bone graft on the single immediate implant placed into healthy fresh extraction socket. There was no difference in implant survival rate, while significant difference on bone density and the bucco-lingual width favor the using bone grafting materials with immediate post-extraction implant. There is insufficient evidence regarding implant stability, marginal bone loss, patient satisfaction.

The two included trials were not adequate to deal with the review objectives. The amount of patients within the individual included studies was small, that will increase the chance of random error and additionally the trials didn't embody most of the outcomes of interest within the review. Currently, there is no agreement on whether or not used of bone grafting materials with the immediately placed implants.

The question of whether the using of bone grafting materials or not can be effected on the survival rate and the stability of immediately placed implants. The results of this review don't permit a strong conclusion relating to the review question. Since two randomized trials were included with small sample sizes, the enclosed trials suffer from serious method limitations that are probably to result in a biased evaluation of the intervention effect, so the quality of evidence rate down by two levels for inexactitude. Additionally the included trials unclear how randomization was performed and the risk of reporting bias were high.

The effectiveness of the bone grafting materials with the immediately placed implants were discussed in systematic review (Esposito et al; 2008). It was in concurrence with our results and concluded that it is unclear whether or not augmentation procedures are required with immediate single implants placed in recent extraction sockets. Chen and Buser, (2014) surveyed the influence of simultaneous bone augmentation procedures on the esthetic outcomes of implants placed in postextraction sites. The review did not find adequate evidence on the effects of bone graft augmentation on the gingival recession or the esthetic outcome of the treatment.

\section{Conclusions:-}

There is inadequate evidence to suggest the bone grafts with immediate post-extraction single tooth implants to enhance implant stability and survival rate. More well-designed RCTs are needed to understand the effectiveness of bone grafting materials with immediate post extraction implant. Such trials should be designed and reported 
according to the Consolidated Standards of Reporting Trials (CONSORT) guidelines (http://www.consortstatement.org).

\section{Disclaimer:-}

The authors declare that they have no conflicts of interest and there was no external funding for the present review.

\section{References:-}

1. Araújo, M. G., Sukekava, F., Wennström, J. L., \& Lindhe, J. (2005). Ridge alterations following implant placement in fresh extraction sockets: an experimental study in the dog. Journal of clinical periodontology, 32(6), 645-652.

2. Botticelli, D., Berglundh, T., Buser, D., \& Lindhe, J. (2003). The jumping distance revisited. Clinical Oral Implants Research, 14(1), 35-42.

3. Bottini, L. P., Ricci, L., Piattelli, A., Perrotti, V., \& Iezzi, G. (2012). Bucco-lingual crestal bone changes around implants immediately placed in fresh extraction sockets in association or not with porcine bone: a non-blinded randomized controlled trial in humans. Journal of periodontology, (0), 1-8.

4. Cardaropoli, G., Araujo, M., \& Lindhe, J. (2003). Dynamics of bone tissue formation in tooth extraction sites. Journal of clinical periodontology, 30(9), 809-818.

5. Chen, S. T., \& Buser, D. (2014). Esthetic outcomes following immediate and early implant placement in the anterior maxilla - a systematic review. Int J Oral Maxillofac Implants, 29(Suppl), 186-215.

6. Chen, S. T., Darby, I. B., Adams, G. G., \& Reynolds, E. C. (2005). A prospective clinical study of bone augmentation techniques at immediate implants. Clinical Oral Implants Research, 16(2), 176-184.

7. Chen, S. T., Darby, I. B., \& Reynolds, E. C. (2007). A prospective clinical study of non-submerged immediate implants: clinical outcomes and esthetic results. Clinical Oral Implants Research, 18(5), 552-562.

8. Christensen, G. J. (1996). Ridge preservation: why not? Journal of the American Dental Association (1939), 127(5), 669-670.

9. De Angelis, N., Felice, P., Pellegrino, G., Camurati, A., Gambino, P., \& Esposito, M. (2011). Guided bone regeneration with and without a bone substitute at single post-extractive implants: 1-year post-loading results from a pragmatic multicentre randomised controlled trial. Eur J Oral Implantol, 4(4), 313-325.

10. Daif, E. T. (2013). Effect of a Multiporous Beta-Tricalicum Phosphate on Bone Density Around Dental Implants Inserted Into Fresh Extraction Sockets. Journal of Oral Implantology, 39(3), 339-344.

11. Esposito, M., Grusovin, M. G., Kwan, S., Worthington, H. V., \& Coulthard, P. (2008). Interventions for replacing missing teeth: bone augmentation techniques for dental implant treatment. Cochrane Database Syst Rev, 3(3).

12. Higgins, J. P., \& Green, S. (Eds.). (2011). Cochrane handbook for systematic reviews of interventions (Vol. 4). John Wiley \& Sons.

13. Hoexter, D. L. (2002). Bone regeneration graft materials. Journal of oral implantology, 28(6), 290-294.

14. Klokkevold, P. R., \& Jovanovic, S. A. (2002). Advanced implant surgery and bone grafting techniques. Carranza's Clinical Periodontology, 9th Edition. Philadelphia: WB Saunders Co, 907-908.

15. Knox, R., Caudill, R., \& Meffert, R. (1991). Histologic evaluation of dental endosseous implants placed in surgically created extraction defects. The International journal of periodontics \& restorative dentistry, 11(5), 364.

16. Misch, C. E., \& Dietsh, F. (1993). Bone-grafting materials in implant dentistry. Implant dentistry, 2(3), 158167.

17. Hassan, K. S., \& Alagl, A. S. (2011). Immediate Dental Implants and Bone Graft. In Implant Dentistry-The Most Promising Discipline of Dentistry. InTech.

18. Schwartz-Arad, D., Gulayev, N., \& Chaushu, G. (2000). Immediate versus non-immediate implantation for fullarch fixed reconstruction following extraction of all residual teeth: a retrospective comparative study. Journal of periodontology, 71(6), 923-928.

19. RevMan, R. (2014). The nordic cochrane centre, the cochrane collaboration. 